\title{
MetaFun: Unveiling sex differences in multiple omics studies through comprehensive functional meta-analysis
}

\author{
Pablo Malmierca-Merlo1, Rubén Sánchez-Garcia1, Rubén Grillo-Risco¹, Irene \\ Pérez-Díez ${ }^{2}$, José F. Català-Senent ${ }^{1}$, María de la Iglesia-Vayá ${ }^{2}$, Marta R. \\ Hidalgo $^{1 *}$, Francisco Garcia-Garcia $^{1^{*}}$
}

\begin{abstract}
${ }^{1}$ Bioinformatics and Biostatistics Unit, Principe Felipe Research Center (CIPF), 46012 Valencia, Spain. ${ }^{2}$ Biomedical Imaging Unit FISABIO-CIPF, Fundación para el Fomento de la Investigación Sanitaria y Biomédica de la Comunidad Valenciana, 46012 Valencia, Spain.
\end{abstract}

*To whom correspondence should be addressed.

\begin{abstract}
Summary: Sex and gender differences in different health scenarios has been thoroughly acknowledged in the literature, and yet, very scarcely analyzed. To fill the gap, here we present MetaFun, which allows to meta-analyze multiple omics datasets with a sex-based perspective, and to combine different datasets to gain major statistical power and to assist the researcher in understanding these sex differences in the diseases under study. Metafun is freely available at bioinfo.cipf.es/metafun Availability and implementation: MetaFun is available under http://bioinfo.cipf.es/metafun. The backend has been implemented in $\mathrm{R}$ and Java and the frontend has been developed using Angular. Contact: fgarcia@cipf.es, mhidalgo@cipf.es

Supplementary information: R code available at https://gitlab.com/ubb-cipf/metafunr
\end{abstract}

\section{Introduction}

The existence of sex and gender differences in different health scenarios has been thoroughly acknowledged in the literature [1,2], and yet, in many cases, not exhaustively analyzed. Many times, the importance of such differences has been neglected, when not denied, and not taken into account in the experimental design of studies. Therefore, most of the underlying reasons for such differences have not been yet established. 
Fortunately, this is beginning to change, and more researchers are including a sex/gender perspective in their scientific approaches. However, generating new data is expensive, and, on the other hand, there exists a huge amount of datasets stored in public and private databases (such as GEO [3] or GDC [4]) which have not been analyzed with a sex/gender perspective. The information in these databases is a powerful tool which should not be wasted.

In order to make the most of this information, multiple studies may be analyzed at the same time. Defined for this purpose, the meta-analysis is a statistical methodology which takes into account the relative importance of different studies in order to combine all of them in a single analysis and extract results based on more evidence and samples [5,6,7]. MetaFun aims to simplify the process and facilitate the access to this methodology to researchers working with omics data which may not be familiar with it, allowing to meta-analyze multiple omics datasets with or without a gender perspective, and to combine them to gain major statistical power and soundness. MetaFun is also a complete suite which allows to analyze transcriptomics data and explore its results at all levels: exploratory analysis, differential expression, pathway analysis, gene set functional enrichment and, of course, the functional meta-analysis.

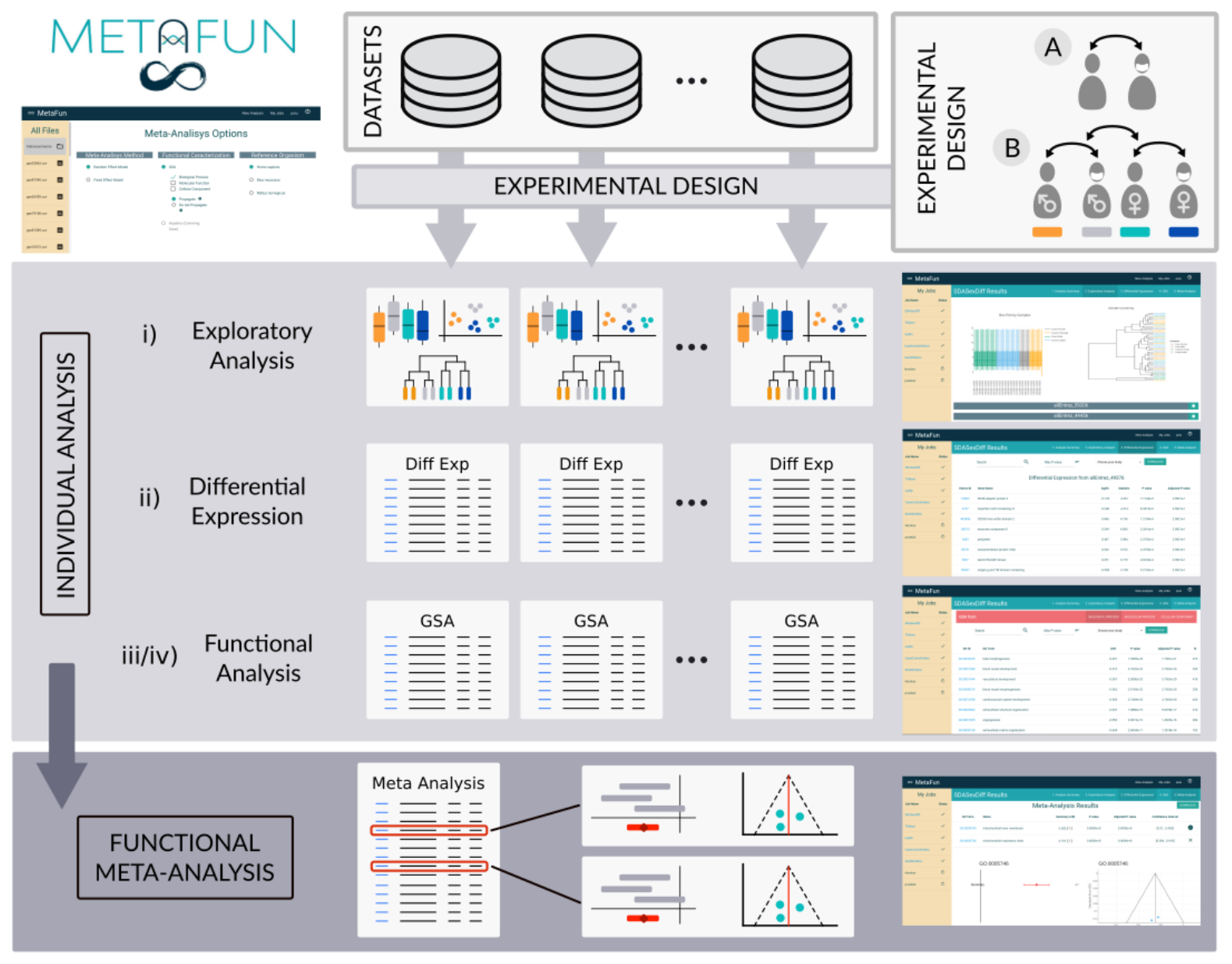

Figure 1: Metafun pipeline. First, datasets must be uploaded and the experimental design selected. Available options include (A) a plain Case vs. Control comparison, and (B) the sex difference 
comparison (Female case vs. Female control) vs. (Male case vs. Male control). Then, individual analysis including i) an exploratory analysis, ii) a gene differential expression and iii) a functional analysis are performed on each dataset separately. Finally, functional results are integrated into a functional meta-analysis. The tool allows the user to explore all results generated in the process.

\section{Methods}

Metafun is available under https://bioinfo.cipf.es/metafun. Help may be found under https://gitlab.com/metafundev/metafun/-/wikis/MetaFun-Help.

\subsection{Input data and experimental design}

MetaFun takes as input a set of at least 2 CSV files including already normalized transcriptomics data which must come from comparable studies with assimilable experimental groups. Accepted reference organisms are, for the moment, human (Homo sapiens), mouse (Mus musculus) and rat (Rattus norvegicus). The selected experimental design must be applicable to all datasets and will be either the plain comparison Case vs. Control (Fig.1A), or the sex specific comparison (Male case vs. Male control) vs. (Female case vs. Female control) (Fig.1B). CSV files including the experimental design information and specifying groups among the samples are accepted, for further assignment of each sample to the available canonical groups (case and control in the simple comparison, and male case, male control, female case and female control in the sex specific one).

\subsection{Individual analyses}

After the selection of the studies and the experimental design, MetaFun analyzes each dataset separately with an individual analysis consisting of: i) an exploratory analysis including boxplots, PCA and cluster plots using plotly library [8], ii) a gene differential expression analysis, using limma package [9], and iii) a Gene Set Enrichment Analysis (GSEA) [10] based on Gene Ontology (GO) [11], from mdgsa package [12]. Figures and tables resulting from these analyses may be explored and downloaded from the Results area once the job is ready. Links to other databases are present to go into detail about the results.

\subsection{Functional meta-analysis}

Finally, MetaFun combines the gene set functional enrichments of all datasets in a single meta-analysis with the same experimental design, using the metafor package [13]. Forest and funnel plots are generated by means of the plot.lyJS library [8]. Figures and tables resulting from this meta-analysis are interactive and may be explored and downloaded from the Results area once the job is ready.

\subsection{Implementation}

MetaFun back-end has been written using Java and $\mathrm{R}$, and is supported by a non relational database (MongoDB [14]) which stores the information of the files, users and launched jobs. 
The front-end has been developed using the Angular Framework [15]. All graphics generated in this webtool have been implemented with Plot.ly [8] except for the exploratory analysis cluster plot which uses ggplot2 package [16].

\subsection{Study Cases}

MetaFun includes as example three sets of pre-selected study cases, one for each accepted species: human, mouse and rat. The study cases can be executed directly from the webtool and allow to explore the functionalities of the tool easily. Human study case includes 9 studies from lung cancer patients [6].

\section{Acknowledgements}

The authors thank the Principe Felipe Research Center (CIPF) for providing access to the cluster, which is co-funded by European Regional Development Funds (FEDER) in Valencian Community 2014-2020.

\section{Funding}

This work was supported by GV/2020/186.

Conflict of Interest: none declared.

\section{References}

1. Ballestri S. et al. NAFLD as a Sexual Dimorphic Disease: Role of Gender and Reproductive Status in the Development and Progression of Nonalcoholic Fatty Liver Disease and Inherent Cardiovascular Risk. Advances in Therapy. 2017; 34: 1291-1326. URL http://link.springer.com/10.1007/s12325-017-0556-1

2. Yuan Y, Liu L, Chen H, et al. Comprehensive Characterization of Molecular Differences in Cancer between Male and Female Patients. Cancer cell. 2016; 29(5): 711-722. doi:10.1016/j.ccell.2016.04.001

3. Barrett T, Wilhite SE, Ledoux P, Evangelista C, Kim IF, Tomashevsky M, Marshall KA, Phillippy KH, Sherman PM, Holko M, Yefanov A, Lee H, Zhang N, Robertson CL, Serova N, Davis S, Soboleva A. NCBI GEO: archive for functional genomics data sets--update. Nucleic Acids Res. 2013 Jan;41(Database issue):D991-5

4. Grossman RL, Heath AP, Ferretti V, Varmus HE, Lowy DR, Kibbe WA, Staudt LM. Toward a Shared Vision for Cancer Genomic Data. New England Journal of Medicine. 2016; 375:12, 1109-1112. doi: 10.1056/NEJMp1607591

5. Casanova Ferrer F, Pascual M, Hidalgo MR, Malmierca-Merlo P, Guerri C, García-García F. Unveiling Sex-Based Differences in the Effects of Alcohol Abuse: A Comprehensive Functional Meta-Analysis of Transcriptomic Studies. Genes. 2020; 11(9):1106. https://doi.org/10.3390/genes11091106

6. Pérez-Díez I, Hidalgo MR, Malmierca-Merlo P, Andreu Z, Romera-Giner S, Farràs R, de la Iglesia-Vayá M, Provencio M, Romero A, García-García F. Functional Signatures in Non-Small-Cell Lung Cancer: A Systematic Review and Meta-Analysis of Sex-Based Differences in Transcriptomic Studies. Cancers. 2021; 13(1):143. https://doi.org/10.3390/cancers13010143

7. Català-Senent JF, Hidalgo MR, Berenguer M, Parthasarathy G, Malhi $H$, Malmierca-Merlo $P$, de la Iglesia-Vayá M, García-García F. Hepatic Steatosis and Steatohepatitis: A Functional 
Meta-analysis of Sex-based Differences in Transcriptomic Studies. Biology of Sex Differences 2021, 12:29; https://doi.org/10.1186/s13293-021-00368-1

8. Plotly Technologies Inc. Collaborative data science. Montréal, QC, 2015. https://plot.ly

9. Ritchie ME, Phipson B, Wu D, Hu Y, Law CW, Shi W, Smyth GK. limma powers differential expression analyses for RNA-sequencing and microarray studies. Nucleic Acids Research. 2015; Volume 43, Issue 7, 20, Page e47, https://doi.org/10.1093/nar/gkv007

10. Subramanian A, Tamayo P, Mootha VK, Mukherjee S, Ebert BL, Gillette MA, Paulovich A, Pomeroy SC, Golub TR, Lander ES, Mesirov JP. Gene set enrichment analysis: A knowledge-based approach for interpreting genome-wide expression profiles, Proceedings of the National Academy of Sciences Oct 2005, 102 (43) 15545-15550; doi: 10.1073/pnas.0506580102

11. Ashburner M, Ball CA, Blake JA, et al. Gene ontology: tool for the unification of biology. The Gene Ontology Consortium. Nat Genet. 2000;25(1):25-29. doi:10.1038/75556

12. Montaner D, Dopazo J. Multidimensional Gene Set Analysis of Genomic Data. PLoS One. 2010; 5(4), e10348. doi: 10.1371/journal.pone.0010348

13. Viechtbauer W. Conducting meta-analyses in $\mathrm{R}$ with the metafor package. Journal of Statistical Software. 2010; 36(3), 1-48. https://www.jstatsoft.org/v36/i03/

14. https://www.mongodb.com/

15. Jain N, Mangal P \& Mehta D. AngularJS: A modern MVC framework in JavaScript. Journal of Global Research in Computer Science. 2014; 5(12), pp.17-23

16. Wickham H. ggplot2: Elegant Graphics for Data Analysis. Springer-Verlag New York. 2016. ISBN 978-3-319-24277-4, https://ggplot2.tidyverse.org 


\section{Supplementary material for}

\section{MetaFun: Unveiling sex differences in multiple omics studies through comprehensive functional meta-analysis}

Pablo Malmierca-Merlo, Rubén Sánchez-Garcia, Rubén Grillo-Risco, Irene Pérez-Díez, José F. Català-Senent, María de la Iglesia-Vayá, Marta R. Hidalgo, Francisco Garcia-Garcia

\section{S1. Webtool overview}

The webtool can be used with an anonymous user or with a registered one. Registered users will keep their data and jobs stored from one session to the other, data and jobs from anonymous users will not be saved after leaving the session. After logging in, the user is directed to the form launching a new job, which can be otherwise accessed through the New Analysis button on the top right part of the tool. The first part of the form includes the user's personal area, in which the user is able to upload and manage the datasets to analyze, and can be otherwise accessed through the user's button on the top right part of the tool. The New Analysis form continues through a series of steps demanding different information which must be filled in. After the launchment and execution of the job, it will be listed on the jobs area, which can be accessed through the My jobs button located on the right top part of the tool, and where all created jobs are stored and can be accessed from to visualize their results.

\section{S2. Input data}

All datasets included in a same meta-analysis should be comparable, including similar experimental designs and individuals with similar conditions. At least two datasets must be included in a meta-analysis. Input data consists of one expression matrix and one experimental design file for each of the datasets in the meta-analysis. The expression matrix must have been normalized, with samples in the columns and EntrezID genes in the rows. The experimental design file must indicate the original group to which each sample belongs, with samples in the rows and groupings in the columns. More than one grouping per file is accepted. Accepted file formats are CSV or TSV for both the expression matrix and the experimental design files. After uploading, expression matrix and experimental design files from the same dataset must be placed in the same row of the data selection form. MetaFun will check that the column names from the expression matrix match with the row names of the experimental design file, and will mark correct matches with a check.

\section{S3. Analysis summary}

After the execution of the job, the Analysis summary tab will show a summary of the main results. This summary includes the selected analysis options (name, contrast a.k.a. experimental design, effect model, functional profile, and reference organism), a table and an interactive barplot describing the number of samples per dataset and per group, a table describing the number of differentially expressed genes in each dataset (per columns, 
number of total analyzed genes, number of significant up-regulated genes, number of significant down-regulated genes, and number of total significant genes), a table with the same columns describing the number of significant functional profile items in each dataset (either enriched functions or differentially activated subpathways, depending on the selected functional profile), and a table with the same columns describing the number of significant functional terms in each ontology (BP for Biological Process, MF for Molecular Functional and $\mathrm{CC}$ for Cellular Component) of the meta-analysis.

\section{S4. Exploratory analysis}

The Exploratory analysis tab contains the figures resulting from the exploratory unsupervised analysis performed on each dataset in the meta-analysis. This analysis includes a boxplot representation of the expression of the samples, a clustering of the samples and a Principal Components Analysis (PCA). The figures include a 2D and a 3D representation of the respectively 2 and 3 first principal components of the PCA, the boxplot and the clustering of the samples. All samples are colored by the experimental design selected in the meta-analysis.

\section{S5. Differential expression}

The differential expression analysis is performed with library limma [9], applying ImFit, contrast.fit and eBayes functions, and taking into account whether the samples are paired or not. Results are displayed as a table in the Differential expression tab of the job once it is ready. The table shows the EntrezID, Gene Name, logarithm of the fold-change (logFC), test statistic, raw p-value and Bonferroni-Holm adjusted p-value of each analyzed feature. The table is initially ordered by the raw p-value, but buttons on the names of the columns allow the user to order the table by each of them. Links from the EntrezID column direct to the $\mathrm{NCBI}$ gene database of the specific gene. Different tools allow the user to search, download and filter the table by a maximum $p$-value.

\section{S6. Gene Set Enrichment Analysis}

The functional analysis consists of a Gene Set Enrichment Analysis (GSEA) [10] based on the Biological Process, Molecular Function and Cellular Component ontologies from Gene Ontology (GO) [11]. The pipeline, performed with the magsa library [12], splits the ontologies, propagates the annotation, filters too generic or too specific annotations, transforms the p-value into an index and performs the corresponding contrasts. Results are displayed as a table in the GSA tab of the job once it is ready. Three sub-tabs show the results separately for the three different ontologies. For each ontology, the table shows the GO ID, GO term, logarithm of the odds-ratio (LOR), raw p-value, Bonferroni-Holm adjusted $p$-value and number of genes included in each analyzed feature. The table is initially ordered by the raw $p$-value, but buttons on the names of the columns allow the user to order the table by each of them. Links from the GO ID column direct to the GO database of the specific term. Different tools allow the user to search, download and filter the table by a maximum p-value. 


\section{S7. Meta-analysis}

The functional meta-analysis integrates the results of the functional analysis and is performed using the rma function in metafor package [13]. For each of the functions, a meta-analysis is carried out that combines the level of overrepresentation (LOR) of that function in the different studies. This will allow us to know whether there is a greater activation of that pathway or biological process in men or in women, for the set of studies evaluated. There are several methods implemented to perform the meta-analysis: fixed effects models (FE) or random effects models (DL DerSimonian \& Laird; HS Schmidt \& Hunter; Hedges, HE) [13]. Results are displayed as a table in the Meta-Analysis tab of the job once it is ready. The table shows the GO ID, GO term, LOR, raw p-value, Bonferroni-Holm adjusted $p$-value and confidence interval of the LOR of each analyzed feature. The table is initially ordered by the raw $p$-value, but buttons on the names of the columns allow the user to order the table by each of them. Links from the GO ID column direct to the GO database of the specific term. Different tools allow the user to search, download and filter the table by a maximum $p$-value.

\section{S8. Study case}

The following case describes the potential use of MetaFun in the characterization of sex differences in lung adenocarcinoma. The results obtained were published in Cancers. 2021 Jan 5;13(1):143. doi: 10.3390/cancers13010143.

\section{Input data:}

For each of the studies we will need two files: a first file with the expression data and a second file with the description of the experimental groups to which each sample belongs, indicating the sex of the participant. In this link you can download the files corresponding to this use case:

https://gitlab.com/ubb-cipf/metafunpipeline/-/blob/master/metafun_sample_data.tar

\section{4 easy steps to launch the meta-analysis job:}

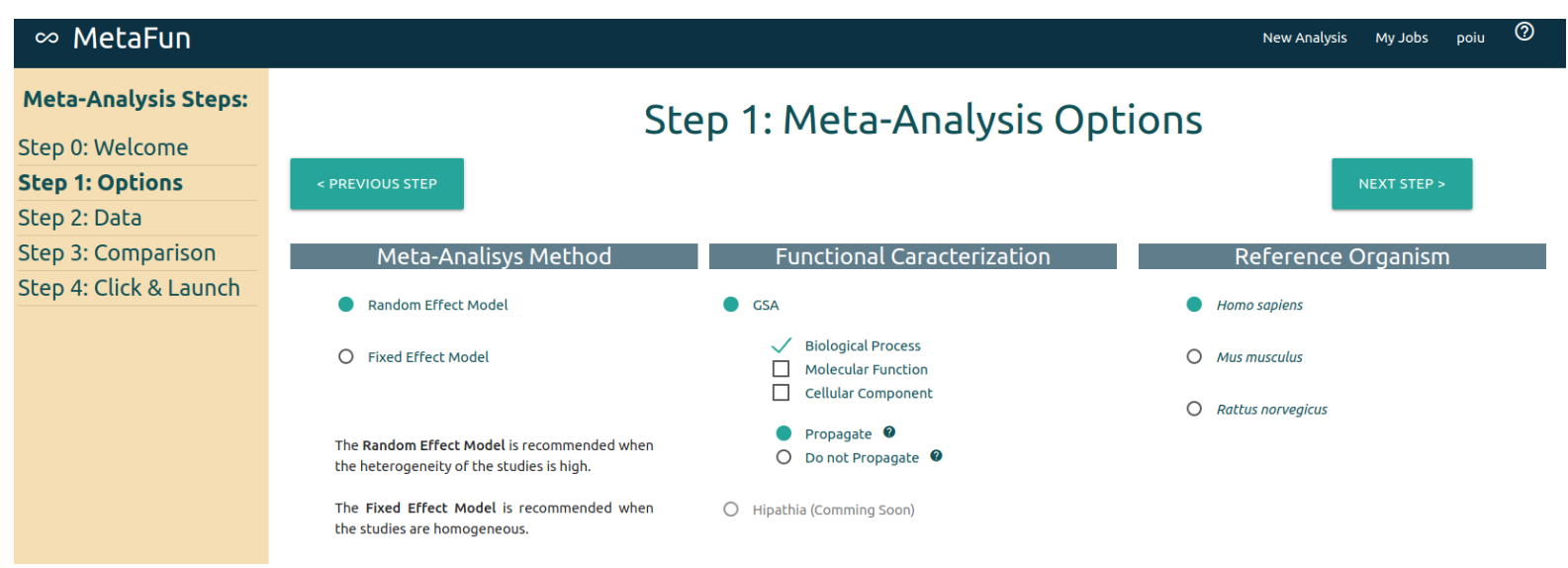


bioRxiv preprint doi: https://doi.org/10.1101/2021.07.13.451905; this version posted July 14, 2021. The copyright holder for this preprint (which was not certified by peer review) is the author/funder, who has granted bioRxiv a license to display the preprint in perpetuity. It is made available under aCC-BY 4.0 International license.

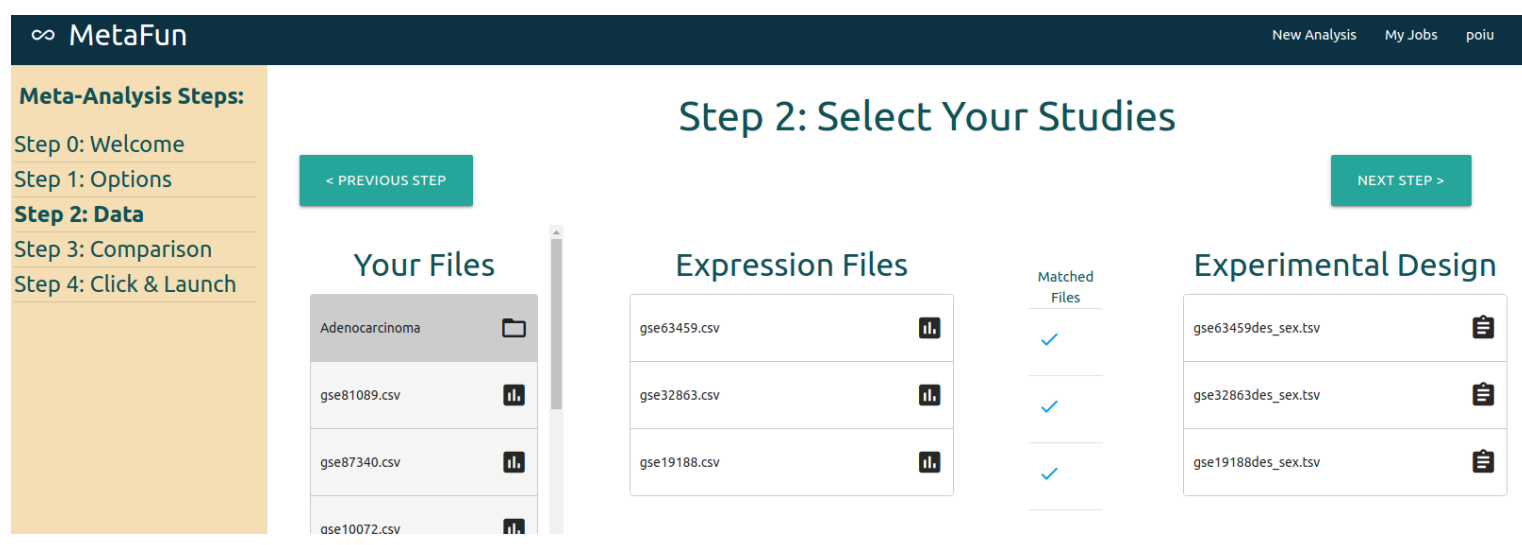

cs MetaFun

Meta-Analysis

Steps:

Step 0: Welcome

Step 1: Options

Step 2: Data

Step 3:

Comparison

Step 4: Click \&

Launch

\section{cs MetaFun}

\section{Step 3: Compare Options}

< PREVIOUS STEP

NEXT STEP >

(Case Female - Control Female) - (Case Male - Control Male)

Csv File

Case Female

Control Female

Case Male

Control Male

gse63459.csv Control_Male

$\checkmark$ Control_Male

$\checkmark$ Control_Male

$\checkmark$ Control_Male

gse32863.csv Adenocarcinoma_Female $\vee$ Adenocarcinoma_Female $\vee$ Adenocarcinoma_Female

Adenocarcinoma_Female

gse19188.csv Control_Male

$\checkmark$ Control_Male

$\checkmark$ Control_Male

$\checkmark$ Control_Male

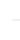

Meta-Analysis Steps:

Step 0: Welcome Step 1: Options Step 2: Data Step 3: Comparison Step 4: Click \& Launch

\section{Step 4: Choose Job's Name \& Launch}

\section{< PREVIOUS STEP}

\begin{tabular}{ll}
\hline \multicolumn{2}{c}{ Selected } \\
\hline Meta-Analysis method: & Random Effect Model \\
\hline Functional Characterization: & GSA \\
\hline Ontologies: & Biological Process \\
\hline Propagate: & YES \\
\hline Reference Organism: & Homo Sapiens \\
\hline
\end{tabular}

Reference Organism: Homo Sapiens

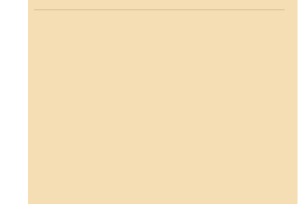


bioRxiv preprint doi: https://doi.org/10.1101/2021.07.13.451905; this version posted July 14, 2021. The copyright holder for this preprint (which was not certified by peer review) is the author/funder, who has granted bioRxiv a license to display the preprint in perpetuity. It is made available under aCC-BY 4.0 International license.

\section{Results:}

For each of the sections described above (1. Analysis Summary, 2. Exploratory Analysis, 3. Differential Expression, 4. Gene Set Analysis, 5. Meta-Analysis), we show the results generated by MetaFun in this use case:

\section{Analysis Summary}

A summary of the results at the different stages of the bioinformatics analysis strategy is detailed:

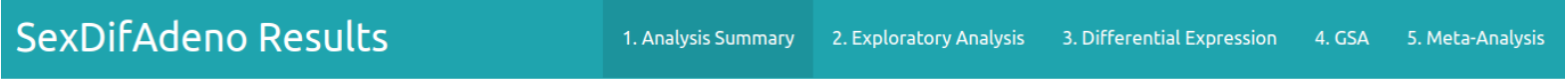

\section{Analysis Summary}

\begin{tabular}{|c|c|c|c|c|c|}
\hline \multicolumn{6}{|c|}{ Job Options } \\
\hline Name & Contrast & Effect Model & Functional Profiling & \multicolumn{2}{|c|}{ Reference Organism } \\
\hline SexDifAdeno & (ME-MS)-(HE-HS) & Random Effect Model & GSA & hsa & \\
\hline \multicolumn{6}{|c|}{ Samples Description } \\
\hline Study Name & Adenocarcinoma Female & Adenocarcinoma Male & Control Female & Control Male & Total \\
\hline gse10072 & 13 & 30 & 8 & 29 & 80 \\
\hline gse19188 & 11 & 21 & 11 & 41 & 84 \\
\hline gse 87340 & 53 & 28 & 8 & 11 & 100 \\
\hline gse32863 & 121 & 105 & 9 & 10 & 245 \\
\hline Total & 198 & 184 & 36 & 91 & 509 \\
\hline
\end{tabular}

\section{SexDifAdeno Results $\quad$ 1. Analysis Summary $\quad$ 2. Exploratory Analysis $\quad$ 3. Differential Expression $\quad$ 4. GSA $\quad$ 5. Meta-Analysis}

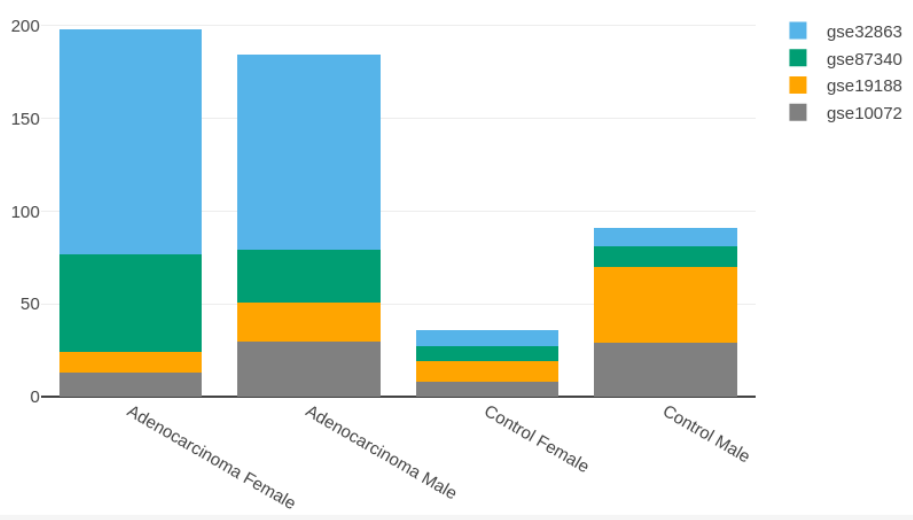


bioRxiv preprint doi: https://doi.org/10.1101/2021.07.13.451905; this version posted July 14, 2021. The copyright holder for this preprint (which was not certified by peer review) is the author/funder, who has granted bioRxiv a license to display the preprint in perpetuity. It is made available under aCC-BY 4.0 International license.

\begin{tabular}{|c|c|c|c|c|}
\hline \multicolumn{2}{|l|}{ SexDifAdeno Results } & $\begin{array}{ll}\text { 1. Analysis Summary } \quad \text { 2. Exploratory Analysis } & \text {. }\end{array}$ & 3. Differential Expression & 4. GSA 5. Meta-Analysis \\
\hline \multicolumn{5}{|c|}{ Differential Expression Summary } \\
\hline Study Name & Total.genes & sig.Total & sig.up & sig. Down \\
\hline gse 10072 & 12993 & 0 & 0 & 0 \\
\hline gse19188 & 20978 & 0 & 0 & 0 \\
\hline gse87340 & 19011 & 3 & 3 & 0 \\
\hline gse32863 & 20978 & 1 & 0 & 1 \\
\hline & Functio & Profiling Summary & & - \\
\hline Study Name & Total.functions & sig.up & sig.Down & sig.Total \\
\hline gse10072 & 7779 & 281 & 547 & 3312 \\
\hline gse19188 & 8298 & 61 & 61 & 2606 \\
\hline gse87340 & 8143 & 42 & 34 & 1854 \\
\hline gse32863 & 8298 & 34 & 22 & 1082 \\
\hline
\end{tabular}

\section{Exploratory Analysis}

Principal component analysis, clustering and boxplots are used to explore the expression levels of each of the samples in the selected studies:

\begin{tabular}{lll|lll}
\hline SexDifAdeno Results & 1. Analysis Summary & 2. Exploratory Analysis & 3. Differential Expression & 4. GSA & 5. Meta-Analysis \\
\hline
\end{tabular}

\section{Exploratory Analysis}

PCA Plot

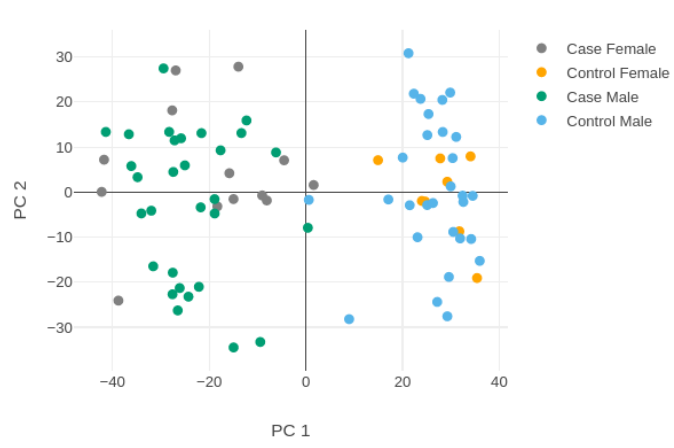

PCA Plot (3D)

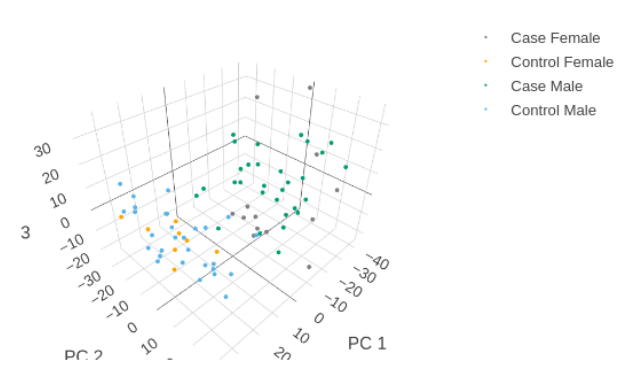


bioRxiv preprint doi: https://doi.org/10.1101/2021.07.13.451905; this version posted July 14, 2021. The copyright holder for this preprint (which was not certified by peer review) is the author/funder, who has granted bioRxiv a license to display the preprint in perpetuity. It is made available under aCC-BY 4.0 International license.

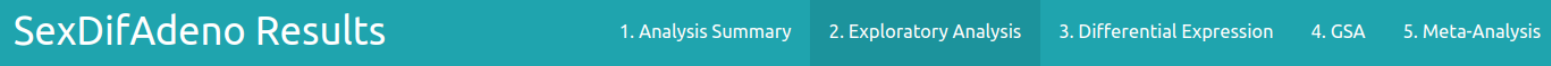
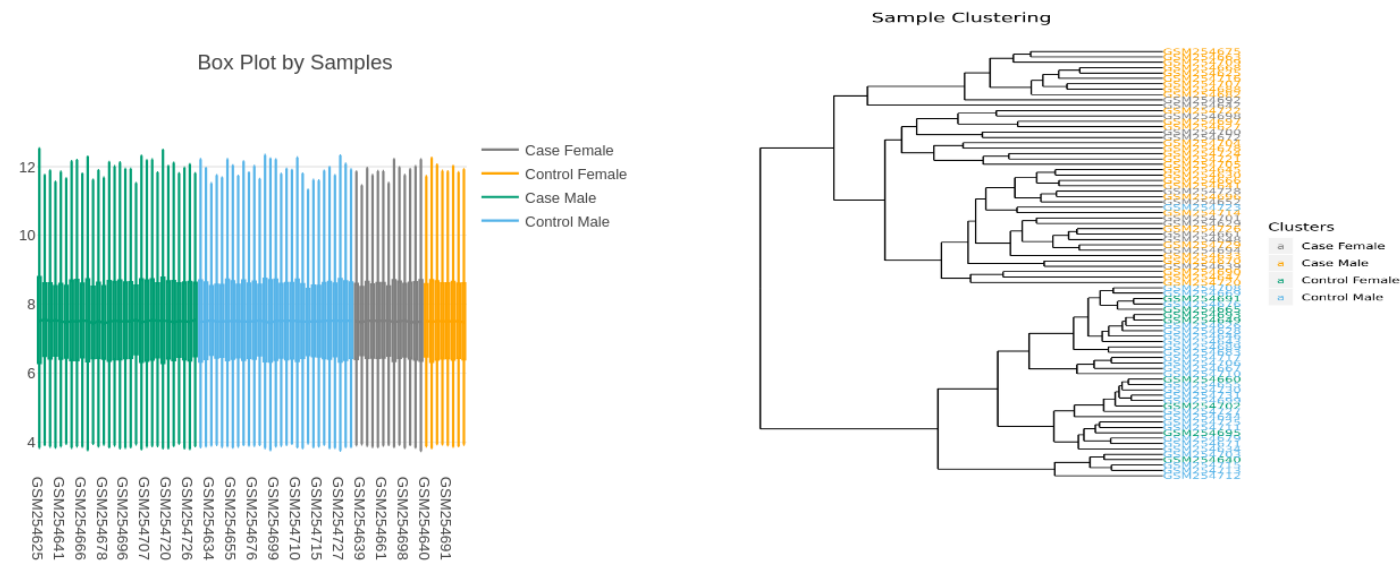

\section{Differential Expression}

For each of the studies, we identified which genes show a differential expression pattern by sex in the disease. By clicking on each of the links to the gene identifiers, you can expand their biological information:

\section{SexDifAdeno Results $\quad$ 1. Analysis Summary $\quad$ 2. Exploratory Analysis $\quad$ 3. Differential Expression $\quad$ 4.GSA $\quad$ 5. Meta-Analysis}

Search $\quad \mathrm{Q} \quad$ Max P-value $\quad=\quad$ Choose your study $\quad$

\section{DOWNLOAD FULL RESULTS}

Differential Expression from gse10072

\begin{tabular}{|c|c|c|c|c|c|}
\hline Entrez ID & Gene Name & $\log \mathrm{FC}$ & Statistic & P value & Adjusted $P$ value \\
\hline 5478 & peptidylprolyl isomerase $A$ & -0.342 & -3.310 & $1.4045 \mathrm{e}-3$ & $9.9848 \mathrm{e}-1$ \\
\hline
\end{tabular}




\section{Gene Set Analysis (GSA)}

Functional characterization of the differential expression results will identify which functions are more active in males and females. The information for each of the significant functions can be expanded by clicking on the link to its identifier.

\begin{tabular}{|c|c|c|c|c|c|c|c|c|c|}
\hline \multirow{2}{*}{\multicolumn{2}{|c|}{$\begin{array}{l}\text { SexDifAdeno Results } \\
\text { GSA from }\end{array}$}} & \multirow[t]{2}{*}{ 1. Analysis Summary } & \multicolumn{2}{|c|}{ 2. Exploratory Analysis } & \multicolumn{2}{|c|}{ Differential Expression } & 4. GSA & \multicolumn{2}{|c|}{ 5. Meta-Analysis } \\
\hline & & & \multicolumn{2}{|c|}{ BIOLOGICAL PROCESS } & \multicolumn{2}{|c|}{ MOLECULAR PROCESS } & \multicolumn{3}{|c|}{ CELLULAR COMPONENT } \\
\hline & Search & Max P-value & $=$ & Choose & your study & $\checkmark$ & \multirow{3}{*}{\multicolumn{2}{|c|}{ Adjusted $\mathrm{P}$ value }} & \multirow[b]{3}{*}{$\mathbf{N}$} \\
\hline & & & \multicolumn{3}{|c|}{ DOWNLOAD FULL RESULTS } & & & & \\
\hline GOID & GO Term & & & & LOR & P value & & & \\
\hline GO:0044772 & mitotic cell cycle phase transition & & & & -0.351 & $9.7362 \mathrm{e}-14$ & & $5.8135 \mathrm{e}-10$ & 474 \\
\hline GO:0043903 & regulation of symbiosis, encompas & assing mutualism through & parasitism & & -0.518 & $5.2450 \mathrm{e}-13$ & & $1.5659 \mathrm{e}-9$ & 200 \\
\hline GO:0050792 & regulation of viral process & & & & -0.515 & $4.2119 \mathrm{e}-12$ & & $8.3831 \mathrm{e}-9$ & 187 \\
\hline
\end{tabular}

\section{$\underline{\text { 5. Meta-Analysis }}$}

Finally in this section, MetaFun shows the functions and pathways that are activated in the set of studies evaluated. If we click on the information icon, we will obtain detailed information on each of these significant functions:

\section{SexDifAdeno Results $\quad$ 1. Analysis Summary $\quad$ 2. Exploratory Analysis $\quad$ 3. Differential Expression $\quad$ 4. GSA $\quad$ 5. Meta-Analysis}

Meta-Analysis Results

\begin{tabular}{|c|c|c|c|c|c|}
\hline GO ID & GO Term & Summary LOR & $P$ value & Adjusted P value & Confidence Interval \\
\hline GO:0000778 & condensed nuclear chromosome kinetochore & $-0.955\left(0^{*}\right)$ & $0.0000 e+0$ & $0.0000 e+0$ & {$[-1.291,-0.619]$} \\
\hline GO:0000779 & condensed chromosome, centromeric region & $-0.507\left(0^{x}\right)$ & $1.0000 \mathrm{e}-2$ & $2.0000 \mathrm{e}-2$ & {$[-0.894,-0.119]$} \\
\hline GO:0000780 & condensed nuclear chromosome, centromeric region & $-0.791\left(0^{x}\right)$ & $0.0000 \mathrm{e}+0$ & $0.0000 \mathrm{e}+0$ & {$[-1.089,-0.493]$} \\
\hline GO:0000793 & condensed chromosome & $-0.360\left(0^{*}\right)$ & $0.0000 \mathrm{e}+0$ & $0.0000 \mathrm{e}+0$ & {$[-0.539,-0.181]$} \\
\hline
\end{tabular}


bioRxiv preprint doi: https://doi.org/10.1101/2021.07.13.451905; this version posted July 14, 2021. The copyright holder for this preprint (which was not certified by peer review) is the author/funder, who has granted bioRxiv a license to display the preprint in perpetuity. It is made available under aCC-BY 4.0 International license.

\section{SexDifAdeno Results $\quad$ 1. Analysis Summary $\quad$ 2. Exploratory Analysis $\quad$ 3. Differential Expression $\quad$ 4. GSA $\quad$ 5. Meta-Analysis}

\section{GO:0098644}
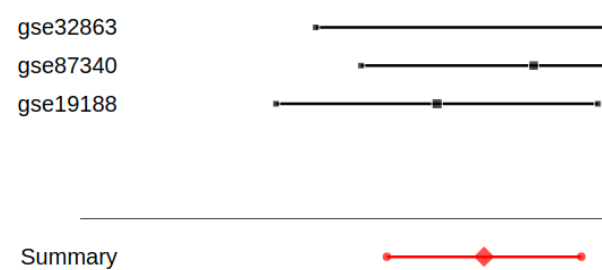

$-0.743\left(\sigma^{*}\right) \quad 0.0000 \mathrm{e}+0$

$0.0000 \mathrm{e}+0$

$[-0.991,-0.495]$

$x$

GO:0098644

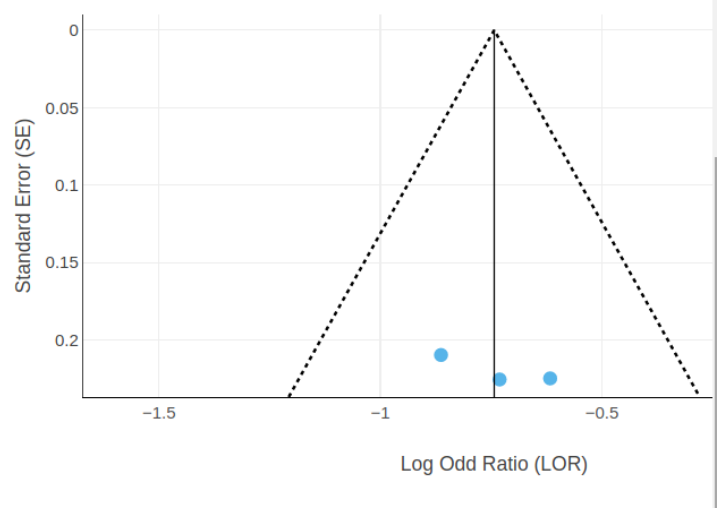

